\title{
The Swiss Corona Stress Study: survey in high school students, March 2021
}

Corresponding author: Dominique de Quervain (dominique.dequervain@unibas.ch)

Co-authors in alphabetical order:

Amanda Aerni

Ehssan Amini

Dorothée Bentz

David Coynel

Christiane Gerhards

Virginie Freytag

Andreas Papassotiropoulos

Nathalie Schicktanz

Thomas Schlitt

Anja Zimmer

Priska Zuber

Affiliation: University of Basel, Transfaculty Research Platform Molecular and Cognitive Neurosciences, Faculty of Psychology and Medical Faculty, Birmannsgasse 8, CH-4055 Basel, Switzerland 


\section{Abstract}

The last survey of the Swiss Corona Stress Study in November 2020 has shown that the proportion of respondents with moderately severe to severe depressive symptoms was $18 \%$, with the youngest group of 14-24 year olds being the most affected at 29\%. Between 8 and 24 March 2021, an additional survey was conducted in the German speaking part of Northwestern Switzerland among 393 high school students with the majority being between 16 and 19 years old. The anonymous survey was completed by $97.5 \%$ of the students. The proportion of respondents with moderately severe to severe depressive symptoms was $27 \%$. In this survey, additional questions were asked about school, social life and vaccination.

The most significant stressor associated with depressive symptoms was perceived school pressure. $46 \%$ of the respondents indicated they were very or extremely stressed because of the pressure of school. Furthermore, the perception that school pressure has increased due to the pandemic (missed material due to closures, quarantine) was strongly correlated with depressive symptoms. Other factors included worries about poorer education or job opportunities and worries about damage to the social network. An additional factor analysis confirmed that stressors related to school build up the factor with the strongest correlation with depressive symptoms (with a large effect size).

\section{Other results}

- $69 \%$ of respondents would get vaccinated if the vaccine were available to them.

- $41 \%$ of respondents would see the change to distance learning as a relief, $46 \%$ as a burden and $13 \%$ neither.

- Only $4 \%$ of respondents said they were not at all afraid of infecting their parents/grandparents or another close person belonging to a risk group.

- $29 \%$ of respondents have at least a moderate to severe fear of suffering from Long-COVID in case of infection. 


\section{Introduction}

The survey of the Swiss Corona Stress Study in November has shown that levels of stress and depressive symptoms have increased significantly during the pandemic in 2020 . The proportion of people with severe depressive symptoms stood at $9 \%$ during lockdown in April, but this figure had risen to $18 \%$ in November [1], [2]. Young people were particularly affected: The prevalence of severe depressive symptoms was $29 \%$ in those aged $14-24,21 \%$ in ages $25-34,17 \%$ in ages $35-44,14 \%$ in ages $45-54,13 \%$ in ages $55-64$ and $6 \%$ in the over 65 s.

In the present survey that was conducted in March 2021, we focused on young people, i.e. high-school students between 14 and 21. To find out more about possible reasons for depressive symptoms we asked additional questions about school, social life, worries and specific measures.

\section{Methods}

We performed an anonymous online survey among pupils of three secondary/high schools (Gymnasium) in North-West Switzerland using the SoSci-Survey server [https://www.soscisurvey.de] provided by sciCORE at the University of Basel [https://scicore.unibas.ch/]. The survey was conducted during regular lessons resulting in a high response rate of $97.5 \%$ (see below). The questionnaire is an adapted version of the Swiss Covid Stress Survey [1], [2] consisting of 113 questions grouped into 48 sections, covering general demographics (sex, age, place of residence, religion, number of siblings, nationality), medical health information (medication, prior psychiatric diseases, COVID-19 infection status). We asked about acceptance of taken federal measures, fears related to the coronavirus, burdens arising from the federal measures, changes in consumption of food / gaming / social media / drugs / sleeping pills and tranquilizers, changed habits, frequency of information seeking about news on the corona crisis, changes in circadian rhythm and eating routines, level of physical activity and meditation. We also asked for worries about age-related issues such as school pressure, distance learning, social networks and worries about the impact on education and future studies and occupation. 
Stress and anxiety levels were assessed using 6-point Likert scales ranging from 0 (not at all) to 5 (extremely strong). Depressive scores were assessed by the "Patient Health Questionnaire 9" - PHQ-9, for the beginning of September and the two weeks prior to the survey. PHQ-9 is a 9-question self-rating questionnaire to screen for the presence and severity of depression. We used a German translation of the PHQ-9 ([3], see [4], [5] for German version).

\section{Statistical analyses}

Comparison of outcomes between school study and Wave 2 subsample:

In order to compare levels of stress and depressive symptoms of the school study participants to levels observed during the second pandemic Wave (November 2020), we extracted from the Swiss Corona Stress Study 2 all participants aged between 14 and 21, who reported to attend high school/secondary school/mandatory school and to live in a German speaking swiss canton. This yielded a total of $N=123$ participants from the second pandemic wave for comparison [2]. Stress levels were compared using the chi-square test. In case of significant difference, post-hoc tests were performed to compare each level between both samples, using both proportion tests and logistic regression accounting for age, gender and existence of prior psychiatric risk.

Depressive symptoms (PHQ-9) levels were compared using both chi-square test (PHQ-9 categories) and linear regression (PHQ-9 total score) accounting for age, gender, and existence of prior psychiatric risk (participants not reporting gender as "male" or "female" were excluded for this analysis).

The proportion of participants showing clinically relevant depressive symptoms (PHQ-9 $\geq 15$ ) were compared using proportion test and logistic regression accounting for age, gender and existence of prior psychiatric risk (participants not reporting gender as "male" or "female" were excluded for this analysis).

\section{Association between outcomes and burden variables:}

The following variables groups were included in analysis: fear of coronavirus (6-items scale from 'Not at all' to 'Very strong'); know someone infected by coronavirus (yes/no); perception of governmental measures adequacy (11-items scale answers grouped into: too loose/adequate/too strict); burden of governmental measures (6-items scale from 'Not at all' to 'Very strong); increased conflicts at home (6-items scale from 'Not at all' to 'Very strong); overwhelmed by switch to digital media (6-items scale from 'Not at all' to 'Very strong); confident 
to overcome the crisis well (6-items scale from 'Not at all' to 'Very strong); personal freedom restricted/fundamental rights violated (6-items scale from 'Not at all to 'Very strong'); change in financial savings (11-items scale answers grouped into: less/unchanged/more); worry by having dinner outdoor without protective measures (6-items scale from 'Not at all' to 'Very strong'); in case of coronavirus positive test would be afraid (...)(6-items scale from 'Not at all' to 'Very strong'); school pressure increased during the pandemic/l feel stressed by current school pressure (6-items scale from 'Not at all' to 'Very strong' ); perception of distance learning (11-items scale from 'Very relieving' to 'Very stressful); facilities of distance learning are good (from 'Not at all' to 'Very strong'); school takes adequate measures towards the pandemic (6-items scale from 'Not at all' to 'Very strong'); worry about the future (education, social network, fewer opportunities)(6-items scale from 'Not at all' to 'Very strong').

Association between burden variables and stress (quantitative score) or depressive symptoms (total PHQ-9 score) were performed using univariate Pearson's correlation tests. Significance level for each outcome was set following a Bonferroni threshold for 41 burden variables tested $(a=0.05 / 41=0.0012)$. Significant associations were also examined under adjustment for age, sex and prior psychiatric disorder (participants not reporting gender as "male" or "female" were excluded for this analysis).

\section{Exploratory factor analysis of burden variables:}

One variable was excluded from analysis due to high missing rate ("Afraid I could suffer from Long-Covid if infected" filled in by $n=247$ participants out of 383). The remaining 40 burden variables were considered for exploratory factor analysis. The number of factors to extract was determined using the fa.parallel function of the $R$ package psych (https://personality-project.org/r/psych/) comparing the scree plot of the observed dataset with that obtained from equally-sized random datasets. A solution with 6 factors was retained. A varimax rotation was applied and subjects' factor scores were derived using the "tenBerge" method.

We further assessed the stability of the derived subjects' factor score by estimating factor scores on subsamples of the initial dataset: the data set was split into five-folds; each fold was successively removed from the dataset and a six factors solution extracted from the remaining four folds. Each time, subjects' factor scores were extracted and correlated with factor scores obtained from the full dataset, yielding an average five-folds estimate of each factor score stability (i.e. correlation). This procedure was repeated 100 times. Average cross-validation 
correlation of the derived factors scores was 0.89 (FA1:0.95; FA2:0.93; FA3:0.90; FA4:0.77 ; FA5:0.94; FA6:0.97).

Variables contributions to factors were interpreted only if they showed a loading $|r|>0.3$ on exclusively one factor.

Association between factors' scores and stress level (quantitative score) or depressive symptoms (total PHQ-9 score) were assessed using linear model regression, accounting for the effects of age, gender and prior psychiatric disorder (participants not reporting gender as "male" or "female" were excluded for this analysis). For each outcome, statistical significance level was set following a Bonferroni threshold for six factors tested $(a=0.05 / 6=0.0083)$.

\section{Results}

\section{Overview}

For our survey of school children we collected data from 08.03.2021 until 24.03.21 at a secondary school (Gymnasium) North West Switzerland. In total, 393 pupils were invited to participate and given time during lessons to fill in our questionnaire; 383 pupils completed the questionnaire, i.e. we have a very high rate of participation (97.5\%), only ten invited persons did not finish our questionnaire. All percentage ratios reported refer to the number of actual participants $(\mathrm{N}=383)$ rather than invitees.

\section{General demographics}

$117(30.5 \%)$ participants were male, $259(67.6 \%)$ female and $7(1.8 \%)$ diverse or not reported. The age ranged from 14 to 21 years (mean 17.4, sd $=1.30$, median 17); most participants reported to have one sibling $(185,48.3 \%)$, two siblings $(97,25.3 \%)$ or no sibling $(56,14.6 \%)$, while $45(11.8 \%)$ participants have three or more siblings.

$325(84.9 \%)$ participants have Swiss nationality, 20 (5.2\%) German nationality. The majority of the participants live in North West Switzerland (363, 94.8\%). 231 (60.3\%) live in a city, 48 $(12.5 \%)$ in agglomeration and $104(27.2 \%)$ in the countryside. $131(34.2 \%)$ participants stated to be religious. 


\section{Medication and health}

$49(12.8 \%)$ participants declared to take medication regularly; several participants reported to suffer from or have suffered from psychiatry diseases: depression 40 (10.4\%) participants, schizophrenia $2(0.5 \%)$ participants, post-traumatic stress disorder 14 (3.7\%) participants, anxiety disorder 29 (7.6\%) participants, obsessive-compulsive disorder 7 (1.8\%) participants and other psychiatric disorders 30 (7.8\%) participants. 14 (3.7\%) participants reported to take psychotropic drugs ('Psychopharmaka').

$17(4.4 \%)$ participants have had an COVID-19 infection without $(3,0.8 \%)$, with mild $(12,3.1 \%)$ or with moderate symptoms (2, 0.5\%). 317 (82.8\%) participants stated they would participate in COVID-19 mass tests at school.

\section{COVID-19 infection-related anxieties}

Almost all participants (355; 92.7\%) know people personally who have had COVID-19 infection and $53(13.8 \%)$ know someone personally who died of the COVID-19 infection.

We asked participants to rate on a scale from not at all (0) to very strongly (5) each of the six questions: I'm afraid that I could get seriously ill with the Corona virus; I'm afraid that I could die of the Corona virus; I'm afraid that someone from the immediate vicinity could become seriously ill with the Corona virus; I'm afraid that someone from the immediate vicinity could die of the Corona virus. The average anxiety scores for these questions were $1.56,0.67,3.18$ and 3.13 respectively, indicating that the anxieties for health and death of others outweighs concerns for the participants themselves. The strongest average anxiety score (3.55) was given for the question I'm afraid that I could infect my parents/grandparents or another close person who belongs to a risk group.

\section{Stress and depressive symptoms: comparison to Wave 2}

We compared levels of stress and depressive symptoms between targeted participants of the School study to the levels observed within a subset of $N=123$ participants with similar age range and living canton (GER) collected during the second pandemic Wave in November 2020 [2](see Methods). 
We observed a significant difference in overall stress levels distribution between both samples (Chi-2 test $p=1.4 \mathrm{e}-02$, Cramer's $V=0.17$ [0.11-0.28]), with a lower fraction of participants from the school study reporting 'Very Strong' stress levels (school study: 14.6\%; wave 2 subsample: $28.5 \%$; proportion test $p=8.4 \mathrm{e}-04$ )(Figure 1A). This difference remained under adjustment for age, gender and prior psychiatric disorder (logistic regression $p=4.5 \mathrm{e}-03$ ).

No significant difference in depressive symptoms (PHQ-9) score was observed between both samples (comparison of distributions Chi-2 test $p=0.12$; linear regression for total PHQ-9 score comparison under adjustment for age, sex and prior psychiatric disorder, $p=0.47$ )(Figure 1B). In addition, no significant difference in the proportion of participants reporting clinically relevant depressive symptoms (PHQ-9 score $\geq 15$ ) was observed between both samples (school study: $26.9 \%$; wave 2 subsample: $31.7 \%$; logistic regression under adjustment for covariates $p=$ 0.65).

\section{Burdens linked to stress and depressive symptoms}

A total of 41 variables assessing the perception of the participants towards adequacy of governmental measures, burden of governmental measures, fear of the coronavirus, school and digital learning burden, and worry about the future were collected.

We first examined the association between each variable and study outcomes.

Stress was most strongly correlated with school pressure variables and worries about the future variables, with large to medium effect sizes respectively (The school pressure has increased during the pandemic: $r=0.52$ [0.45;0.59], $p=1.7 e-28$; Worry that $I$ will get worse education because of the pandemic: $r=0.38$ [0.29;046], $p=8.1 \mathrm{e}-15)$ (Figure 2A).

Stress also showed a significant association, yet of smaller effect size, with increased conflicts at home $(r=0.26[0.17 ; 0.35], p=1.7 e-07)($ Figure $2 A)$.

In addition, small effect sizes associations were observed between stress and a few variables linked to burden of governmental social restrictions (for instance The restrictions in social life burden me: $r=0.27$ [0.17;0.36], $p=8.3 e-08$ ) as well as fear of the virus (for instance Afraid that I could infect my parents/grandparents or another close person who belongs to a risk group: $r=$ $0.24[0.14 ; 0.33], p=2.9 \mathrm{e}-6)($ Figure $2 \mathrm{~A})$. 
Similarly to stress, higher depressive symptoms were mostly correlated with school pressure variables and worries about the future with large to medium effect sizes (The school pressure has increased during the pandemic: $r=0.51$ [0.43;0.58], $p=2.1 \mathrm{e}-26$; Worry that I will get worse education because of the pandemic: $r=0.47$ [0.39;0.55], $p=6.7 \mathrm{e}-23$ )(Figure 2A). In addition, we also observed a significant correlation between depressive symptoms and increased conflicts at home $(r=0.3$ [0.21;0.39], $p=1.7 e-09)$. A small effect size association was also observed between depressive symptoms and lower financial reserve $(r=-0.21,[-0.31 ;-0.12], p$ $=2.3 e-05)$. In addition, small effect sizes associations were observed with burden of non availability of vaccination $(r=0.2$ [0.09;0.3], $p=1.9 \mathrm{e}-04)$, and burden of wearing a mask $(r=$ 0.18 [0.07;0.27], $p=7 e-04$ ), although we note that corresponding lower bounds of effect sizes confidence intervals are lower than $|0.1|$.

Finally, higher stress and higher depressive symptoms were associated with lower confidence to overcome the crisis well, with medium effect sizes (depressive symptoms: $r=-0.41$ [-0.49;-0.32], $p=3.8 \mathrm{e}-17$; stress: $r=-0.32$ [-0.41;-0.23], $p=1.5 \mathrm{e}-10)$ (Figure 2).

Comparable associations patterns were observed under adjustment for age, gender and prior psychiatric disorder (Figure 2B).

The burden variables collected are measuring partially overlapping concepts and hence do show some level of inter-correlations. Hence, in order to disentangle the main factors associated with stress or depressive symptoms, we performed an exploratory factor analysis of the collected burden variables.

A total of six factors was extracted (see Methods), accounting for about $40 \%$ of total variation in the dataset. In order to interpret independently the association of each factor with stress or depressive symptoms, a varimax rotation was applied thus yielding uncorrelated subjects' factor scores.

Examination of the variables contributing the most to each factor (Figure 3) indicated that the factor FA1 is mainly characterized by variables corresponding to increased school pressure and worries about the future. The second factor FA2 is mainly characterized by variables linked to the perception of the adequacy of governmental measures (measures rated too strict). Variables loadings on the third factor FA3 point to increased feeling of burden by governmental restrictions. The factors FA4 and FA5 are mainly characterized by variables linked to fear of the 
coronavirus, for relatives and oneself, respectively. Finally the sixth factor FA6 shows high loadings for variables linked to perception or adequacy of digital learning facilities.

In sum, the extracted factors do recapitulate main uncorrelated contributions from the overall set of collected burden variables.

We next tested each factor for association with the stress and depressive symptoms respectively.

Stress correlated significantly with the factor FA1 corresponding to increased school burden and worries about the future (linear model association accounting for age, gender and prior psychiatric disorder: $r=0.45$ [0.37;0.53], $p=9 \mathrm{e}-20$, after Bonferroni adjustment for six factors tested). Association of lower effect size was also observed between stress and factor FA3 reflecting increased feeling of burden by governmental restrictions (linear model association accounting for age, gender and prior psychiatric disorder: $r=0.23[0.13 ; 0.32], p=5.4 \mathrm{e}-5$, after Bonferroni adjustment for six factors tested)(Figure 4).

Depressive symptoms showed exclusively significant correlation with the school burden-worries about the future factor FA1 (linear model association accounting for age, gender and prior psychiatric disorder: $r=0.57$ [0.5;0.63], $p=5.6 \mathrm{e}-33$, after Bonferroni adjustment for six factors tested)(Figure 4). 


\section{Figures}

\section{Figure 1}

A. Comparison of stress levels between this survey (school) and the wave 2 subsample (November 2020)

Proportion is given relative to the total number of participants within each study (school $\mathrm{N}=383$; wave 2 $\mathrm{N}=123$ ). Error bars correspond to $95 \%$ confidence intervals.

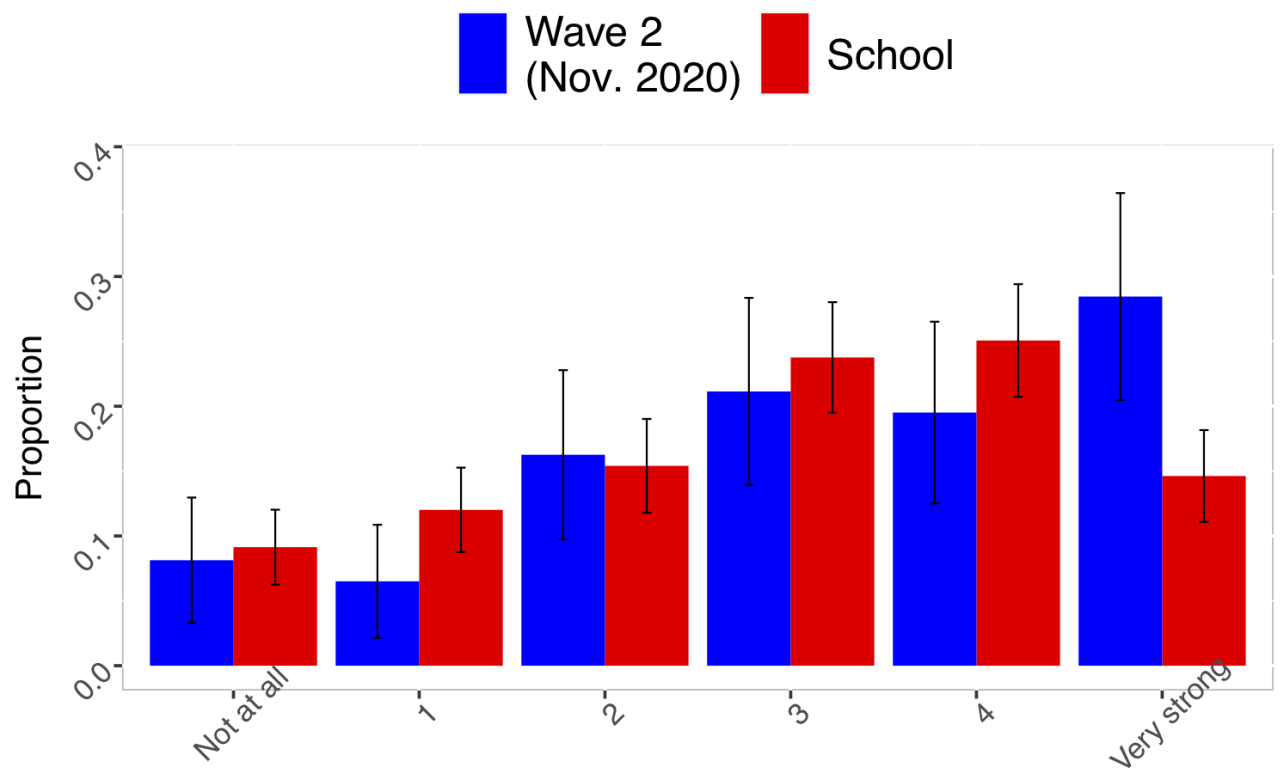

Stress 
B. Comparison of PHQ-9 depressive symptoms between this survey (school) and the wave 2 subsample (November 2020)

Proportion is given relative to the total number of participants within each study (school $\mathrm{N}=383$; wave 2 $\mathrm{N}=123$ ). Error bars correspond to $95 \%$ confidence intervals.

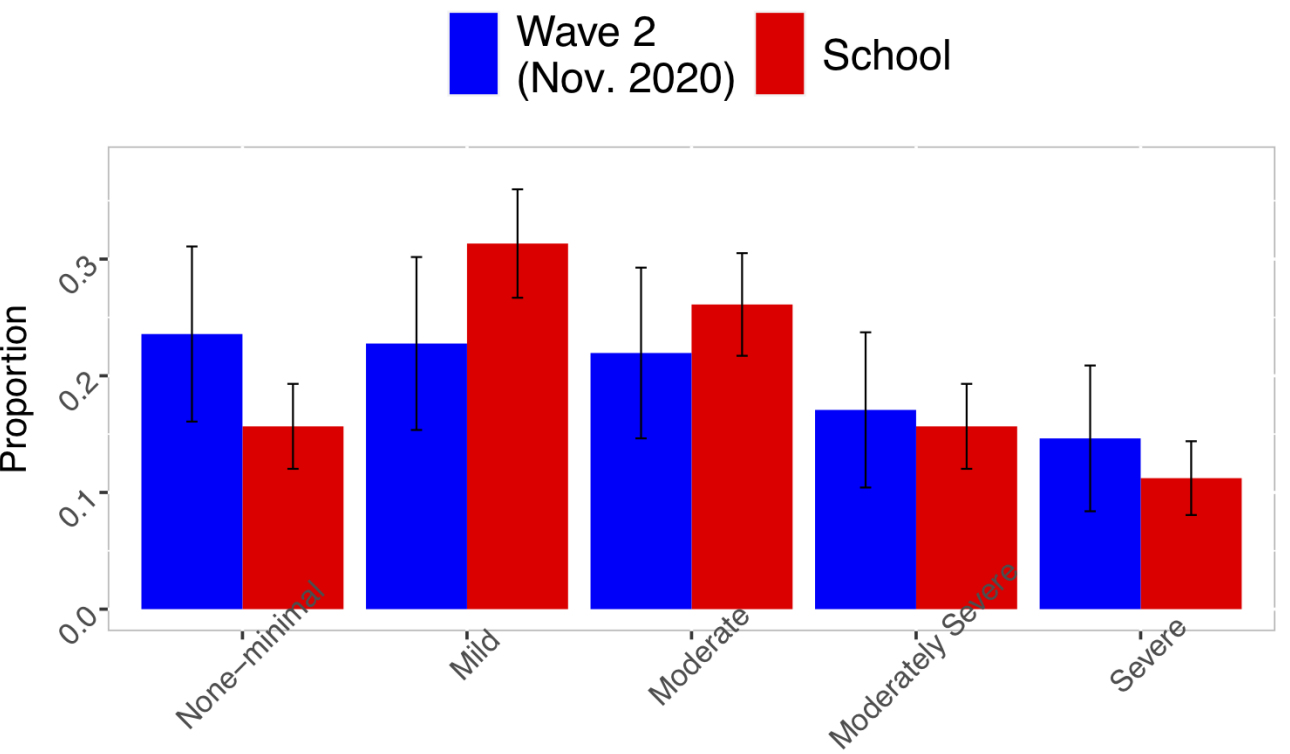

PHQ 


\section{Figure 2}

\section{A. Association between burden variables and stress (left panel) or depressive symptoms} (right panel).

Vertical axis: burden variables labels. Labels are colored by main categories:

Green: variables linked to school burden;

Purple: variables referring to worries about future;

Orange: burden of governmental restrictions;

Brown: fear of coronavirus;

Cyan: perception of adequacy of governmental measures.

Horizontal axis: $r$ effect size for each association. Error bars correspond to $95 \%$ confidence intervals. Colored scheme based on effect size amplitude: grey: association not statistically significant $(p>0.05$ after Bonferroni adjustment for 41 variables tested). Black: significant association with small effect size ( $|r|$ $>0.1)$; Blue: significant association with medium effect size $(|r|>0.3)$; Red: significant association with large effect size $(|r|>0.5)$.

Vertical dashed lines indicate $|r|=0.1$ effect size. 


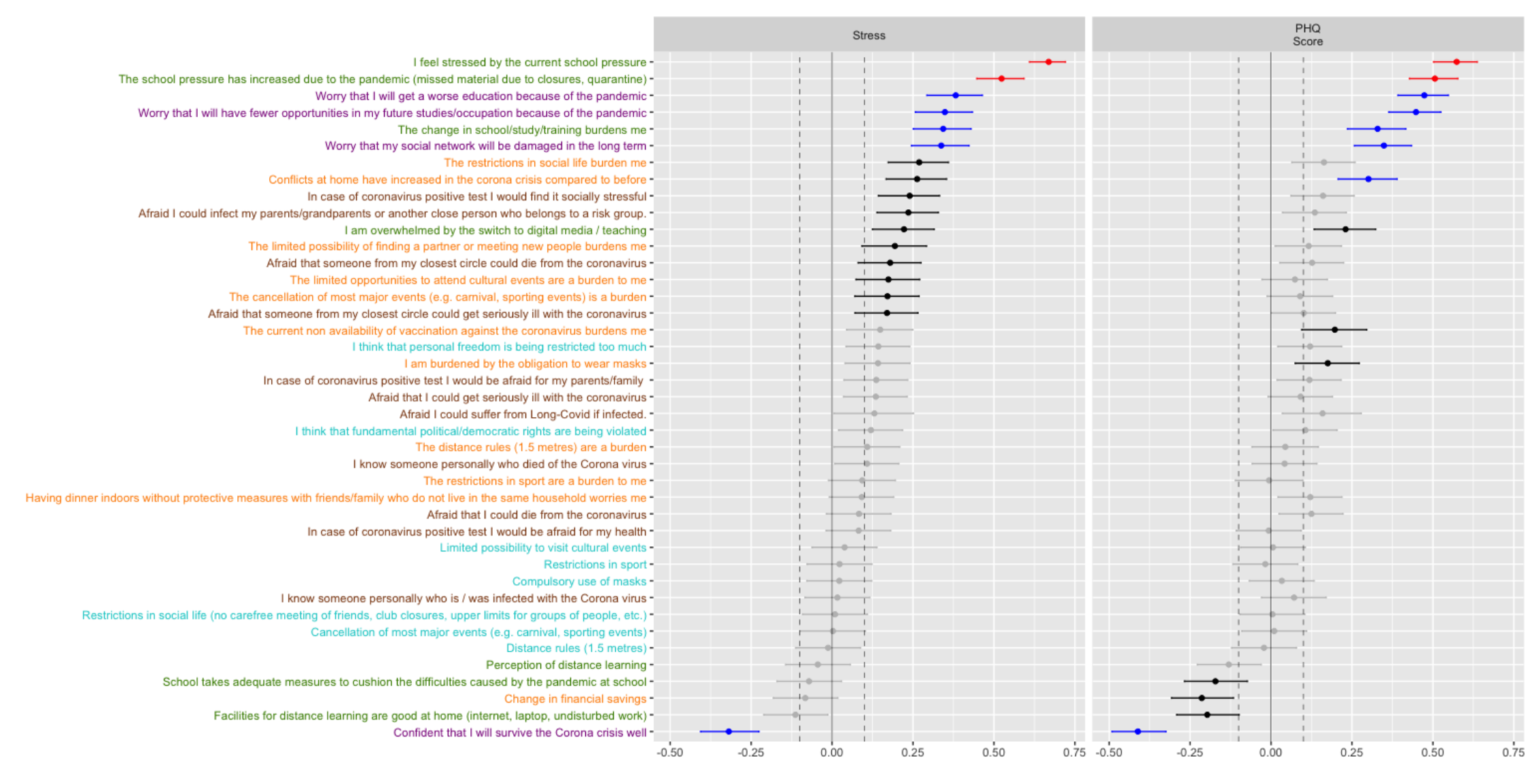




\section{B. Association between burden variables and stress (left panel) or depressive symptoms (right panel) under adjustment for age, gender and prior psychiatric disorder.}

Vertical axis: burden variables labels. Labels are colored by main categories:

Green: variables linked to school burden;

Purple: variables referring to worries about future;

Orange: burden of governmental restrictions;

Brown: fear of coronavirus;

Cyan: perception of adequacy of governmental measures.

Horizontal axis: $r$ effect size for each association under adjustment for age, gender and prior psychiatric disorder. Error bars correspond to $95 \%$ confidence intervals. Colored scheme based on effect size amplitude: grey: association not statistically significant ( $p>0.05$ after Bonferroni adjustment for 41 variables tested). Black: significant association with small effect size $(|r|>0.1)$; Blue: significant association with medium effect size $(|r|>0.3)$; Red: significant association with large effect size $(|r|>$ $0.5)$.

Vertical dashed lines indicate $|r|=0.1$ effect size. 


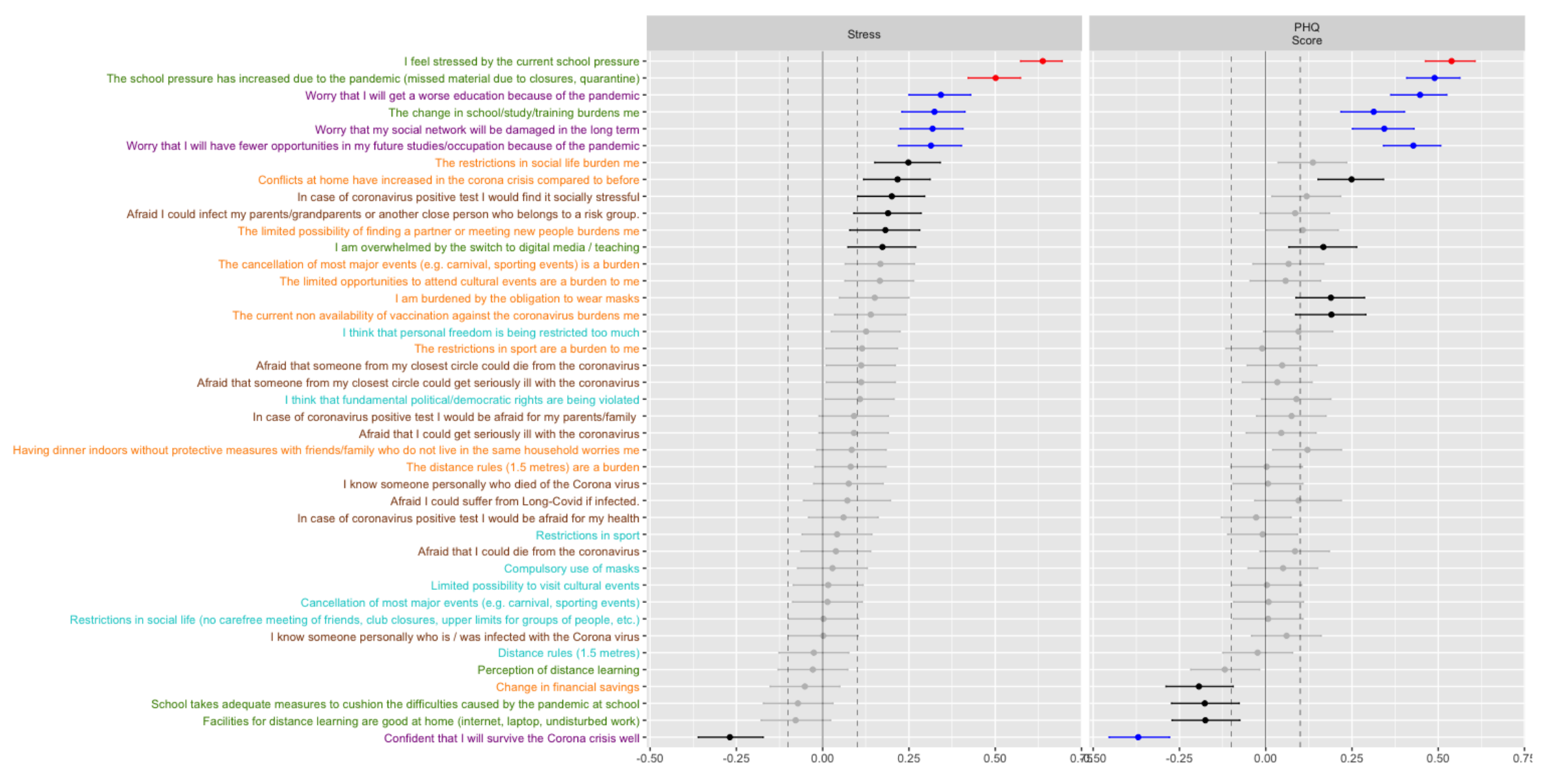




\section{Figure 3}

\section{Variables loadings on each of the six factors extracted from exploratory analysis of burden variables.}

Only variables showing a loading $|r|>0.3$ were interpreted.

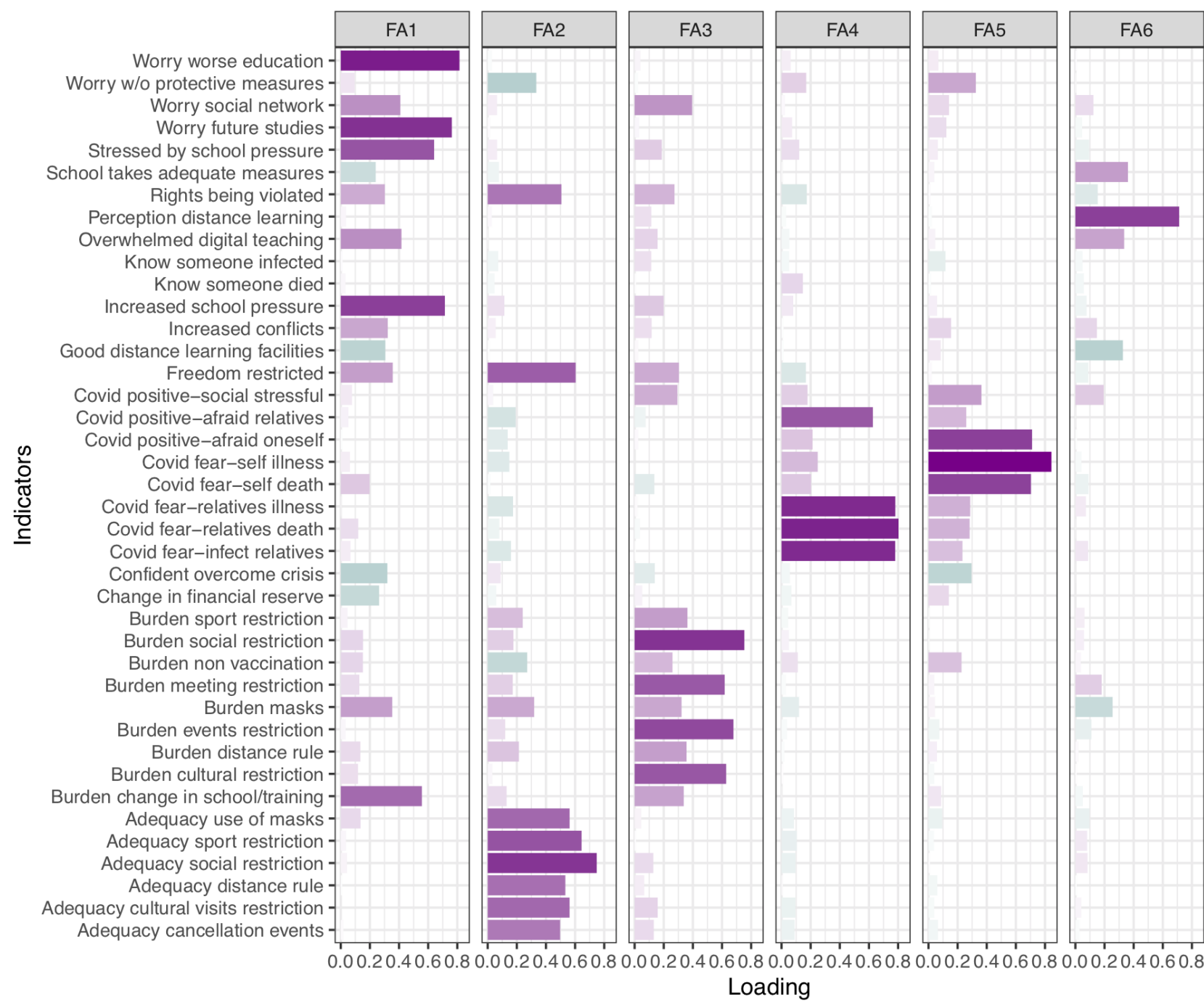




\section{Figure 4}

\section{Effect sizes $r$ of association between factor scores and stress (left panel) or depressive symptoms (right panel).}

Horizontal axis: $r$ effect size for each association. Error bars correspond to $95 \%$ confidence interval. Colored scheme based on effect size amplitude: grey: association not statistically significant $(p>0.05$ after Bonferroni adjustment for 6 variables tested). Black: significant association with small effect size $(|r|$ $>0.1)$; Blue: significant association with medium effect size $(|r|>0.3)$; Red: significant association with large effect size $(|r|>0.5)$.

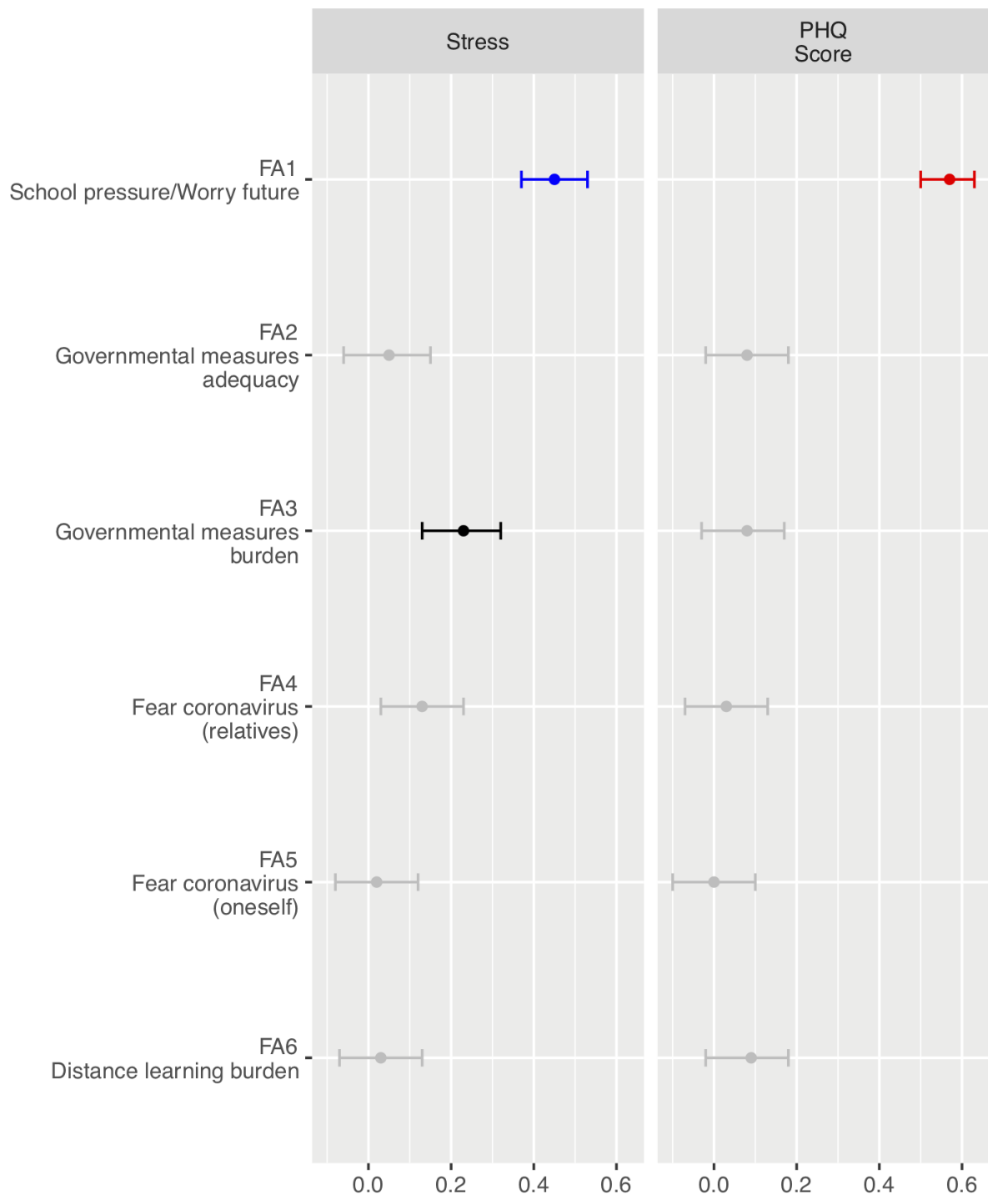




\section{References}

[1] D. de Quervain et al., "The Swiss Corona Stress Study," OSF Preprints, Apr. 2020. doi: 10.31219/osf.io/jqw6a.

[2] D. de Quervain et al., "The Swiss Corona Stress Study: second pandemic wave, November 2020." OSF Preprints, Dec. 16, 2020, doi: 10.31219/osf.io/6cseh.

[3] K. Kroenke and R. L. Spitzer, "The PHQ-9: A New Depression Diagnostic and Severity Measure," Psychiatric Annals, vol. 32, no. 9, pp. 509-515, Sep. 2002, doi:

10.3928/0048-5713-20020901-06.

[4] K. Gräfe, S. Zipfel, W. Herzog, and B. Löwe, "Screening psychischer Störungen mit dem "Gesundheitsfragebogen für Patienten (PHQ-D)“," Diagnostica, vol. 50, no. 4, pp. 171-181, Oct. 2004, doi: 10.1026/0012-1924.50.4.171.

[5] B. Löwe, R. L. Spitzer, S. Zipfel, and W. Herzog, PHQ-D : Gesundheitsfragebogen für Patienten; Manual Komplettversion und Kurzform, 2. Pfizer, Karlsruhe, 2002. 